\title{
Pharmacogenetics from ethno-cultural perspectives
}

\author{
Chee Ng and David Castle
}

\section{Summary}

Advances in genetic technology have focussed on how genetic polymorphisms may impact on drug metabolism, response, adverse effects and clinical outcome. As drug handling and response is significantly driven by genetic factors, genotyping holds promise for personalised medicines. However, high genetic variability and multiple drug effects present a significant challenge to provide useful guidelines in clinical practice. In addition, the expressions of polymorphic genes that control neurobiological functions and drug metabolising

enzymes are also influenced by environmental and dietary factors. With the increasing accessibility and affordability of genotyping, predicting clinical efficacy and adverse effects in individuals from diverse backgrounds holds greater potential. Further research into the pharmacogenetic differences from cross-ethnic perspectives may increase our understanding of the effects of genetic variants on drug efficacy and tolerability. This is likely to enable more rational psychopharmacotherapy that can minimize adverse effects, enhance efficacy, improve adherence and save costs in the long term.

SL J Psychiatry 2010; 1 (2):29-31

\section{Introduction}

It is well known that people respond differently to medications both in terms of efficacy and side effects. Clinicians cannot predict these differences reliably but can be guided by various factors such as biological, physical and ethnic differences. Understanding the genetic variants that predict how individuals respond to different medications can guide optimal pharmacotherapy. This essay outlines some current understandings in this area, with a particular emphasis on ethnic influences.

\section{Pharmacogenetics and drug response}

While pharmacogenetics is defined as the study of variability in drug handling and response due to genetic variations between individuals (1), the term is inter-changeable with pharmacogenomics which refers arbitrarily to the study of the sum of different genes that determine drug behaviour within a population. The clinical utility of pharmacogenetics on drug targets and metabolism of psychotropic drugs have received much attention due to recent advances in molecular genetics, not least the mapping of the human genome.

Variants ofdifferentgenotypes thatcontrol the expression of drug metabolising enzymes, drug receptors, and drug transport systems have a profound influence on both pharmacokinetics and pharmacodynamics (2). Among the various components (absorption, distribution, metabolism and excretion), metabolism is regarded as the most significant factor in determining interindividual and inter-ethnic differences. The metabolism of psychotropic medications (including antipsychotics, antidepressants and anxiolytics) are predominantly mediated by cytochrome $\mathrm{P} 450$ isoenzymes (CYP) especially CYP2D6, CYP2C19, CYP1A2 and CYP3A4. Hence, variability in drug metabolism is related to the genotype variants of these drug metabolising isoenzymes. It has long been recognised that differences among ethnic groups in the functioning of these enzymes lead to variability in drug response to psychotropic agents (3).

Four metabolic phenotype groups results from genes that control the expression of metabolic activity: (i) normal or extensive metabolisers (EMs) who have normal to high metabolic activity; (ii) poor metabolisers (PMs) who have low to absent metabolic activity; (iii) intermediate metabolisers (IMs) also have impaired metabolic function, which is greater than PMs but less than EMs; and (iv) ultrarapid metabolisers (UMs) who have extreme metabolic activity leading to rapid metabolism and excretion of drugs. As there is a lower metabolic clearance rate of substrates in PMs, they have higher risk of toxicity from medications. On the contrary, UMs who have a high clearance of substrates may be under-dosed with medications leading to treatment failure.

The CYP2D6 gene is highly polymorphic and has over 70 variant alleles but only a handful of these alleles are common, accounting for over $95 \%$ of the polymorphisms in humans (2). Duplications of the CYP2D6 gene results in UMs who have 2 or more functional genes. Genetic polymorphisms also occur with CYP2C19 and homozygous deficient variants $(* 2 / * 2, * 2 / * 3$ or *3/*3) are associated with CYP2C19 PMs. Recently, the CYP2C19*17 allele which is associated with UM phenotype, has been found to show lower serum level of escitalopram in corresponding subjects (4). By taking a single blood sample it is possible to genotype individuals to determine the particular metabolic profile traits. Although the expressions of these genes can be influenced by non-genetic factors, these functional polymorphisms are largely responsible for the differences in metabolic capacity, and consequently clinical response and adverse effects. 


\section{Ethnic differences in pharmacogenetic variants}

The influences of ethnicity in the clinical responses to and metabolism of antidepressants have been reported $(5,6)$. Differences in metabolic activity based on ethnicity are also evident, with Asians having an increased likelihood of carrying genetic polymorphisms of CYP enzymes that reduce drug clearance rates (7). Genetic studies of CYP2C19 have found that $15-30 \%$ of certain Asian populations are PMs compared to 3-6\% of Caucasians and 2-4\% of Africans (5). This is most likely due to the high prevalence of CYP2C19*2 and CYP2C19*3 variant in Asians which is less prevalent in other populations (8). For CYP2D6 enzyme, up to half of Asians carry CYP2D6 genotypes with intermediate functioning allele that results in impaired metabolic capacity $(5,9)$. In contrast, the prevalence of UMs is relatively high (up to $29 \%$ ) in certain African populations.

While aldehyde dehydrogenase and alcohol dehydrogenase deficiencies in East Asian populations are well known, ethnic variations in the frequencies of genetic polymorphisms involving other drug metabolising enzymes also exist. For instance, $\mathrm{N}$-acetyltransferase PM rates in Caucasian populations is 38-50\% while in Asians and Eskimos it is about 10$25 \%$ (10). The high rate of fast acetylators in Asians may have implications for the metabolism of drugs such as nitrazepam, clonazepam and possible phenelzine (11).

Pharmacogenetics also applies to the genetic subtypes of drug receptors or targets that can determine response to a particular drug. The serotonin transporter (5HTT) is a prime target of action of many antidepressants, notably selective serotonin reuptake inhibitors (SSRIs) (6). A widely studied polymorphism occurs in the promoter region and is referred to as the long (l) and short (s) allele of the serotonin transporter gene (5HTTLPR). The presence of the 1 allele $(1 / 1$ or $1 / \mathrm{s})$ is associated with a higher density of 5-HT transporters and may affects response to specific antidepressants (6). The s/s genotype of the 5-HTTLPR gene in Caucasians is associated with poorer response to SSRIs compared to those carrying the 1 allele. However, Asian patients with the same genotype have been reported to show the opposite pattern of response $(12,13)$.

Polymorphisms also exist in the promoter region of the noradrenaline gene (NET T-182C) which targets the noradrenergic system. In a Japanese study, the presence of a T allele in NET T-182C was associated with a superior response to dual-acting serotonin and noradrenaline, while the A/A genotype of NET G1287A conferred a slower response to therapy (14). In another study of subjects of Korean ethnicity, there was a strong association between response to nortriptyline in individuals homozygous for the $\mathrm{G}$ allele in NET G1287A (15).

\section{Implications of pharmacogenetics in clinical practice and sociocultural context}

Given the ethnic variations in pharmacogenetics, extrapolating clinical drug trial results obtained in one ethnic group to all populations can no longer be done uncritically. Standard psychotropic dosage recommendations (one size fits all approach) clearly do not apply to all population groups. Caution has to be exercised when applying international drug guidelines based on evidence (often derived from western populations) to specific populations where no such data has been gathered. However, it is also inappropriate to base treatment decisions on ethnicity alone as there are marked inter-individual differences in metabolism even within the same ethnic group. Rather, we need to consider how ethnic factors can best inform management decisions. According to $\mathrm{Ng}$ et al (5),“...ethnicity remains a useful and important clinical consideration in pharmacotherapy, which like other variables, such as age, gender, hepatic/renal function, weight and physical status, cannot be disregarded in tailoring individual dosage of medication".

Hence, dose adjustments according to genotypes/ phenotypes may be necessary to take account of differences in metabolic capacity (16). The diversity of polymorphism across ethnic groups results in differences in metabolic phenotype among subjects from different ethnic groups. This has important implications for clinical practice both in different countries as well as in multicultural communities within single countries. The higher rate of extrapyramidal side effects associated with neuroleptic use among Asian subjects is an example of such drug sensitivities (11). As a result of lower tolerance to medication, medication adherence rates may be poor. Thus, lower doses of medication are frequently needed for improved clinical efficacy and safety in Asian populations, together with lower starting doses and slower dose increments (17). Genotyping can guide clinicians in individually tailoring drug dosages for optimal efficacy and minimising adverse effects with differential dosing for different metabolic genotypes. This is particularly important for psychotropic drugs with a narrow therapeutic range where excessive dosage regimens could lead to toxic side effects and adverse drug interactions.

Dietary habits and the use of traditional medicinal herbs, which are largely influenced by sociocultural factors, also have significant implications for pharmacotherapy. Culture may determine the type and amount of foods consumed, the way they are prepared and the exposure to environmental chemicals or pesticides used in agriculture. Socially sanctioned behaviour may affect the level of consumption of alcohol, cigarettes, caffeine or illicit substances within a cultural group. All these factors can have profound effects on the metabolism of many substrates by CYP1A2, CYP3A4 and possibly other isoenzymes.

In addition, local customs can influence the use of traditional and alternative medicines as a preference or in addition to Western medicines. Differences in dietary practices and the use of herbal medicines in various ethnic groups may affect both pharmacokinetics and pharmacodynamics of psychotropics. The diet and food effects on drug metabolism are even more relevant because dietary habits are changing rapidly with globalisation. As non-Western countries become more developed, many are adopting a Western style diet (e.g. 
"fast food"), and traditional herbs are gaining popularity in Western countries. However, dietary factors are yet to be given adequate attention and almost invariably are not considered in clinical drug studies.

Sociocultural beliefs about disease and specific drugs can also affect drug treatment responses, often in more potent ways than biological mechanisms (18). These include beliefs and expectations of drug therapeutic and side effects, treatment adherence, placebo effects and use of herbal treatments. Perceptions and the subjective experience of psychiatric drugs are also associated with treatment adherence and response. Identifying negative attitudes and perception within the clinical context may improve therapeutic alliance, compliance and treatment completion. Assessment of attitudes towards medications that are influenced by cultural beliefs and views are hence informative for treatment optimisation in different cultures.

\section{Declaration of interest}

The authors have no conflict of interest to declare.

Associate Professor Chee Ng, MB BS, MMed, MD, FRANZCP,Department of Psychiatry, University of Melbourne, Director, International Unit, St Vincent's Mental Health; and Deputy Director, Professorial Unit,

The Melbourne Clinic

Professor David Castle, MB ChB, MSc, MD, MRCPsych, FRANZCP Chair of Psychiatry, St Vincent's Hospital, University of Melbourne.

Corresponding author

Chee Ng, St Vincent's Mental Health, PO Box 2900,

Fitzroy, Victoria, Australia 3065

Email:cng@unimelb.edu.au

\section{References}

1. Evans WE, Relling MV. Pharmacogenomics: translating functional genomics into rational therapeutics. Science 1999; 286: 487-491.

2. Meyer UA. Pharmacogenetics and adverse drug reactions. Lancet 2000; 356: 1667-1671.

3. Lin KM, Poland, RE, Wan YY, Smith M, Lesser IM. The evolving science of pharmacogenetics: clinical and ethnic perspectives. Psychopharmacology Bulletin 1996; 32: 205-217.

4. Rudberg I et al. Impact of ultrarapid CYP2C19*17 allele on serum concentration of escitalopram in psychiatric patients. Clin Pharmacol Ther 2008;83(2):322-327.
5. $\mathrm{Ng} \mathrm{CH}$ et al. The emerging role of pharmacogenetics: implications for clinical psychiatry. ANZ Psychiatry 2004;38(7):483-489.

6. Lee MS. Pharmacogenetics of ethnic populations. In: $\mathrm{Ng} \mathrm{CH}$, Lin KM, Singh B, Chiu E. editors. Ethnopsychopharmacology: Advances in current practice. New York, Cambridge University Press; 2008.

7. Lambert T, Norman T. Ethnic differences in psychotropic drug response and pharmacokinetics. In: Ethnopsychopharmacology: Advances in current practice. In: $\mathrm{Ng} \mathrm{CH}$, Lin KM, Singh B, Chiu E. editors. New York, Cambridge University Press; 2008.

8. Goldstein JA, Ishizaki T, Chiba K, et al. Frequencies of the defective CYP2C19 alleles responsible for the mephenytoin poor metabolizer phenotype in various Oriental, Caucasian, Saudi Arabian and American black populations. Pharmacogenetics 1997;7:59-64.

9. Binder E, Holsboer F. Pharmacogenetics an antidepressant drugs. Ann Med 2006;38:82-94.

10. Grant DM, Morike K, Eichelbaum M, Meyer UA. Acetylation pharmacogenetics. J Clin Invest 1990; 85: 968-972.

11. Lin KM, Poland RE, Smith MW, Strickland TL, Mendoza R. Pharmacokinetic and other related factors affecting psychotropic responses in Asians. Psychopharmacology Bulletin 1991; 27: 427-438.

12. Lesch KP, Gutknecht L. Pharmacogenetics of the serotonin transporter. Prog Neuropsychopharmacol Biol Psychiatry 2005;29:1062-1073.

13. Ng C, Easteal S, Tan S, Schweitzer I, Ho B, Aziz S. Serotonin transporter polymorphisms and clinical response to sertraline across ethnicities. Progress in Neuropsychopharmacology and Biological Psychiatry 2006;30: 953-957.

14. Yoshida $\mathrm{K}$ et al. Prediction of antidepressant response to milnacipran by norepinephrine transporter gene polymorphisms. Am J Psychiatry 2004;161:1575-1580.

15. Kim $\mathrm{H}$ et al. Monoamine transporter gene polymorphisms and antidepressant response in Koreans with late-life depression. JAMA 2006;296(13):1609-1618.

16. Kirchheiner J, Brosen K, Dahl ML, Gram LF, Kasper S, Roots I, Sjoqvist F, Spina E, Brockmoller J. CYP2D6 and CYP2C19 genotype-based dose recommendations for antidepressants: a first step towards subpopulationspecific dosages. Acta Psychiatr Scand 2001; 104:173192.

17. Lin KM, Smith MW, Ortiz V. Culture and Psychopharmacology. Psychiatr Clin North Am 2001; 24: 523-537.

18. Ng C, Klimidis S. Cultural factors and the use of psychotropic medications. n: Ethnopsychopharmacology: Advances in current practice. In: $\mathrm{Ng} \mathrm{CH}$, Lin KM, Singh B, Chiu E. editors. New York, Cambridge University Press; 2008. 